\title{
Mapeamento de locos de características quantitativas para desempenho no cromossomo 4 de suínos ${ }^{1}$
}

\author{
Kleibe de Moraes Silva ${ }^{2}$, Simone Eliza Facioni Guimarães ${ }^{3}$, Paulo Sávio Lopes ${ }^{3}$, Carlos \\ Souza do Nascimento ${ }^{3}$, Marcos Soares Lopes ${ }^{3}$, Mayara Morena Del Cambre Amaral ${ }^{3}$
}

\author{
${ }^{1}$ Fonte de financiamento: CNPq, CAPES e FAPEMIG \\ 2 Pós-graduação em Zootecnia - UFV. \\ ${ }^{3}$ Departamento de Zootecnia, Universidade Federal de Viçosa, Viçosa, Minas Gerais, CEP: 36570-000.
}

RESUMO - Uma população F2 de suínos obtida a partir do intercruzamento da geração F1, proveniente do acasalamento divergente de dois machos da raça naturalizada brasileira Piau com 18 fêmeas comerciais (Landrace $\times$ Large White $\times$ Piétrain) e genotipada para cinco marcadores tipo microssatélite foi utilizada com o objetivo de mapear locos de características quantitativas associados a características de desempenho no cromossomo 4. As características avaliadas foram: número de tetas, peso ao nascimento, peso aos 21, 42, 63, 77 e 105 dias de idade, peso ao abate, consumo de ração, conversão alimentar e ganho de peso médio diário dos 77 aos 105 dias de idade e idade ao abate. Utilizou-se o método de regressão por intervalo de mapeamento com análises realizadas por meio do programa QTL EXPRESS. Verificou-se a presença de apenas um QTL significativo (para peso aos 77 dias). A utilização deste QTL na Seleção Assistida por Marcadores deve ser feita depois que a posição desse QTL for refinada, com possível identificação da mutação causal e estimação de seus efeitos.

Palavras-chave: cruzamento divergente, marcadores microssatélite, melhoramento animal

\section{Mapping of quantitative trait loci for performance on chromosome 4 in pigs}

\begin{abstract}
The objective of this work was to map QTL on porcine chromosome 4 and associate them to performance traits. For this, a F2 pig population was established from a cross from the F1 generation, produced by crossing two naturalized Brazilian Piau sires and 18 commercial dams (Landrace $\times$ Large White $\times$ Piétrain). The population was genotyped for five microsatellite markers. The performance of traits evaluated in the population were: teat number, birth weight, weight at 21, 42, 63, 77 and 105 days of age, slaughter age, feed intake, feed gain ratio, average daily gain from 77 to 105 days of age, and slaughter age. Data were analyzed through multiple regressions developed for analysis of interval mapping using QTL EXPRESS software. Significant evidence for QTL was verified for weight at 77 days of age. The use of this information for marker assisted selection can be done after fine mapping, identification of the causative mutation and estimation of its effects.
\end{abstract}

Key Words: animal breeding, divergent cross, microsatellite markers

\section{Introdução}

Ao contrário da pressuposição de que as características quantitativas eram controladas por muitos genes de pequeno efeito, Lande (1981) sugeriu que poucos genes poderiam ser responsáveis por grande proporção da variação fenotípica das características quantitativas. Esses locos foram denominados locos de características quantitativas ou QTL (Geldermann, 1975) e podem ser identificados por meio de cruzamentos entre raças divergentes utilizando marcadores genéticos espalhados ao longo do genoma. As primeiras evidências de QTL em suínos foram identificadas por Andersson et al. (1994) no cromossomo 4, no qual foi encontrado QTL controlando taxa de crescimento e espessura de toucinho, responsáveis, respectivamente, por 12 e $17 \%$ da variabilidade fenotípica. Após estes achados, vários programas de mapeamento genético em suínos utilizando raças exóticas têm permitido a identificação de QTL controlando características de crescimento, reprodutivas e de qualidade de carne (Bidanel \& Rothschild, 2002).

Segundo Pereira (2001), a medida mais eficiente para avaliar o crescimento é o peso ou o ganho de peso em determinada fase da vida do animal. Desta forma, têm sido incluídas nas metas de seleção características de crescimento em certas idades; e as informações de genes e QTL 
encontrados podem ser utilizadas em conjunto aos métodos tradicionais de seleção, por meio da Seleção Assistida por Marcadores (MAS), para melhorar a eficiência de seleção. Em comparação aos métodos tradicionais de seleção, um ganho genético adicional de $64 \%$ pode ser obtido utilizando-se as informações moleculares juntamente com os métodos tradicionais (Henshall \& Goddard, 1997). Apesar de as características de crescimento apresentarem herdabilidades de moderada a alta (Paszek et al., 1999), algumas limitações da seleção baseada no fenótipo poderiam ser parcialmente eliminadas com o uso da informação molecular, o que possibilitaria a seleção diretamente pelo genótipo (DNA), resultando em seleção mais acurada, precoce e de baixo custo (Dekkers \& Hospital, 2002). Para a implementação da MAS, os QTL devem ser identificados e seus efeitos estimados. O objetivo ao realizar este trabalho foi mapear QTL associados às características de desempenho no cromossomo 4 de suínos a partir de uma população F2 obtida de um cruzamento divergente.

\section{Material e Métodos}

A formação da população e a obtenção dos dados fenotípicos foram realizadas na Granja de Melhoramento de Suínos do Departamento de Zootecnia da Universidade Federal de Viçosa (UFV), em Viçosa, MG, Brasil, no período de novembro de 1998 a julho de 2001. Para obtenção do desequilíbrio da fase de ligação entre os marcadores e os QTL, foi utilizado o delineamento F2. Para formação da geração F1, 18 fêmeas de linha comercial (Landrace $\times$ Large White $\times$ Piétrain) desenvolvida na UFV e selecionada para características de desempenho foram inseminadas com sêmen de dois varrões da raça naturalizada brasileira Piau. Entre os animais F1, foram selecionados ao acaso 11 machos e 54 fêmeas, provenientes de diversas leitegadas, que foram intercruzados por monta natural para obtenção da geração F2. Os animais F2 foram pesados ao nascimento e desma- mados aos 21 dias de idade. Após o desmame, todos os animais receberam ração comercial à vontade e, aos 10 dias de idade, os machos foram castrados. Dos 77 aos 105 dias de idade, os animais foram mantidos em baias individuais e o consumo de ração foi medido diariamente para cálculo da conversão alimentar. Os animais submetidos ao teste foram pesados no início e ao final do teste para cálculo do ganho de peso diário. Após o teste, os animais foram alojados em baias e abatidos assim que atingiam $65 \mathrm{~kg}$ $(64,7 \pm 5,6)$, aproximadamente $147,9 \pm 10,6$ dias de idade.

A análise genotípica foi conduzida no Laboratório de Biotecnologia Animal (LABTEC) do Departamento de Zootecnia da UFV. O DNA dos animais parentais, F1 e F2, foi extraído de sangue coletado imediatamente após o abate utilizando-se o método de extração por precipitação com sal (Miller et al., 1988), mantendo-as soluções (na concentração aproximada de $25 \eta \mathrm{g} / \mu \mathrm{L}$ ) a $4^{\circ} \mathrm{C}$. Os animais foram previamente genotipados para o gene Halotano, uma vez que as características de desempenho são influenciadas pela ação deste gene (Band et al., 2005).

Os animais foram genotipados utilizando-se cinco marcadores tipo microssatélite (SW489, S0301, S0001, S0217 e SW58). Para as amplificações, utilizaram-se termocicladores MJ Reseach, Inc. modelo PTC-100/96. As reações constituíam-se de Taq polimerase $1 \mathrm{U}$, dNTPs $0,2 \mathrm{mM}$, primers $0,2 \mathrm{mM}$ cada, Tris- $\mathrm{HCl} \mathrm{pH} 8,320 \mathrm{mM}$, $\mathrm{KCl} 50 \mathrm{mM}$. As concentrações de magnésio $\left(\mathrm{Mg}^{2+}\right)$ variaram de 2 a 4 mM, em decorrência do padrão de amplificação

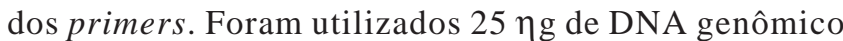
por reação, em um volume final de $20 \mu \mathrm{L}$. As amplificações dos fragmentos dos marcadores foram observadas em gel de poliacrilamida a $5 \%$ e coradas com nitrato de prata, com aplicação de $5 \mu \mathrm{L}$ de cada reação. Informações adicionais sobre a descrição dos marcadores utilizados e suas especificações (Tabela 2) podem ser obtidas no endereço eletrônico: http://www.marc.usda.gov/genome/swine/ swine.html. Os produtos da reação em cadeia da polimerase

Tabela 1 - Número de observações, média, desvio-padrão e valores mínimos e máximos para as características estudadas

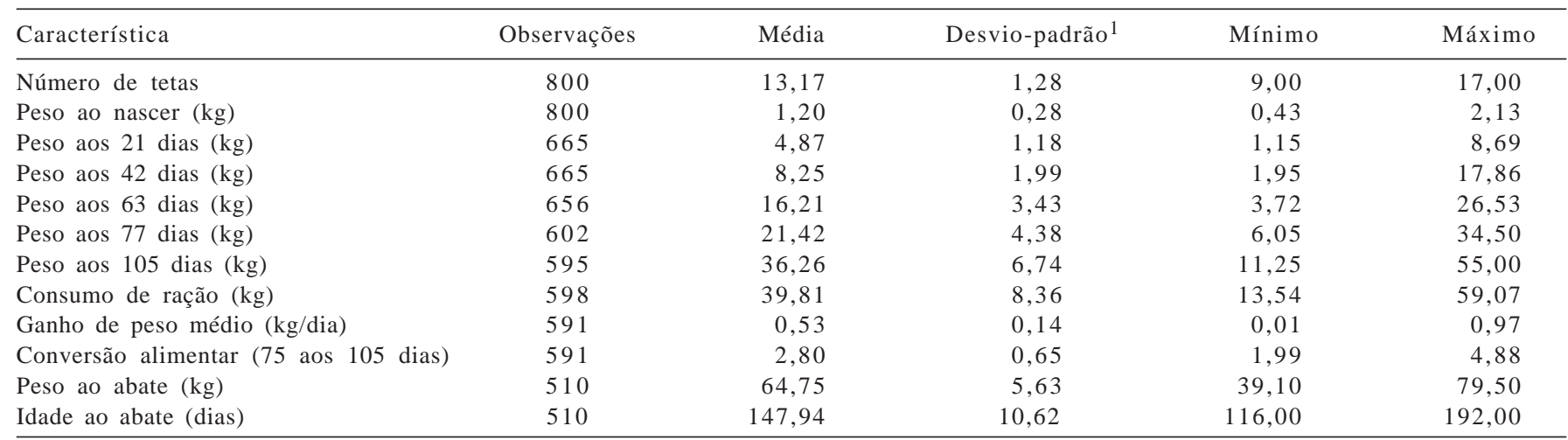


Tabela 2 - Marcadores utilizados na varredura do cromossomo 4

\begin{tabular}{lccc}
\hline Marcador & Posição $^{1}$ & Fluoróforo & $\mathrm{T}\left({ }^{\circ} \mathrm{C}\right)^{2}$ \\
\hline SW498 & 0,0 & HEX & 55 \\
S0301 & 33,0 & FAN & 58 \\
S0001 & 59,2 & HEX & 50 \\
S0217 & 112,0 & FAN & 62 \\
SW58 & 152,3 & TE T & 58 \\
\hline
\end{tabular}

${ }^{1}$ Posição em cM para o mapa médio obtido com o programa CRIMAP (v.2.4) para esta população.

${ }^{2}$ Temperatura de anelamento em ${ }^{\circ} \mathrm{C}$.

(PCR) foram submetidos à análise de fragmentos por meio de seqüenciador automático ABI PRISM 310 (Applied Biosystems) utilizando-se o programa GENESCAN (Applied Biosystems) para a classificação dos fragmentos amplificados. O cálculo do Conteúdo de Informação Polimórfica (PIC) para cada marcador, a heterozigosidade esperada (He) e a heterozigosidade observada (Ho) foram obtidos por meio do programa CERVUS v.3.0 (Marshall et al., 1998) (Tabela 3). O mapa de ligação foi constituído utilizando-se o programa CRIMAP, versão 2.4 (Green et al., 1990), com a opção "build".

O mapeamento de QTL foi realizado por meio do programa QTL EXPRESS para população F2 (Seaton et al., 2002) (http://www.qtl.cap.ed.ac.uk) foi construído utilizando-se as distâncias do mapa médio entre os sexos (Tabela 2). Segundo Haley et al. (1994), assume-se que o QTL é dialélico, com alelos alternativos fixados em cada raça parental. Considerou-se o genótipo QQ para o genótipo homozigoto da raça naturalizada brasileira Piau com efeito $a$, qq para o genótipo homozigoto da raça Comercial com efeito - $a$ e Qq para o heterozigoto com efeito $d$. A probabilidade de cada indivíduo F2 apresentar cada um dos três genótipos do QTL foi calculada conforme os marcadores a intervalos de 1cM ao longo do cromossomo. Essas probabilidades foram utilizadas para a regressão das características nos coeficientes aditivos e de dominância do QTL em estudo para cada animal. No cálculo da fração da variância aditiva explicada pelo QTL, utilizou-se a equação $h_{Q}^{2}=a^{2} / 2 \sigma_{y}^{2}$ (Perez-Enciso

Tabela 3 - Marcadores microssatélites utilizados na varredura do cromossomo 4

\begin{tabular}{lccccc}
\hline Marcador & Posição $^{1}$ & PIC $^{2}$ & HExp. $^{3}$ & HObs. $^{4}$ & N $^{\circ}$ de alelos \\
\hline SW489 & 0,0 & 0,32 & 0,58 & 0,68 & 3 \\
S0301 & 33,0 & 0,50 & 0,52 & 0,56 & 3 \\
S0001 & 59,2 & 0,50 & 0,60 & 0,60 & 3 \\
S0217 & 112,0 & 0,41 & 0,57 & 0,73 & 3 \\
SW58 & 152,3 & 0,42 & 0,56 & 0,69 & 4 \\
\hline
\end{tabular}

${ }^{1}$ Posição em cM para o mapa médio para esta população.

2 Conteúdo de Informação Polimórfica.

3 Heterozigosidade esperada.

${ }^{4}$ Hererozigosidade observada. et al., 2000), em que $a$ representa o efeito aditivo do QTL e $\sigma$, o desvio-padrão fenotípico.

Os efeitos aditivos e de dominância do QTL foram estimados pelo seguinte modelo estatístico:

$$
\mathrm{Y}_{\mathrm{ijkl}}=\mathrm{S}_{\mathrm{i}}+\mathrm{L}_{\mathrm{j}}+\mathrm{H}_{\mathrm{k}}+\left(\mathrm{C}_{\mathrm{ijkl}}-\mathrm{C}\right) \mathrm{b}+\mathrm{c}_{\mathrm{a}} \mathrm{a}+\mathrm{c}_{\mathrm{d}} \mathrm{d}+\mathrm{e}_{\mathrm{ijkl}}
$$

em que: $\mathrm{Y}_{\mathrm{ijkl}}=$ fenótipo; $\mathrm{S}_{\mathrm{i}}=$ efeito fixo do sexo $\mathrm{i}, \mathrm{i}=1,2$; $\mathrm{L}_{\mathrm{j}}=$ efeito fixo do lote $\mathrm{j}, \mathrm{j}=1,2,3,4,5 ; \mathrm{H}_{\mathrm{k}}$ = efeito fixo do genótipo do gene Halotano k, k=1(NN), $\left.2(\mathrm{Nn}) ; \mathrm{C}_{\mathrm{ijkl}}-\mathrm{C}\right) \mathrm{b}=$ ajustamento para as co-variáveis tamanho de leitegada ao nascimento para peso ao nascimento; tamanho de leitegada ao desmame para P21, P42, P63, P77, P105 e PA; peso aos 77 dias para consumo de ração, ganho de peso e conversão alimentar; $\mathrm{e}_{\mathrm{ijkl}}=$ resíduo.

Os valores $\mathrm{C}_{\mathrm{a}}$ e $\mathrm{C}_{\mathrm{d}}$ são funções das probabilidades condicionais do QTL, dado o genótipo dos marcadores, e são calculados da seguinte forma:

$\mathrm{C}_{\mathrm{a}}=\mathrm{P}\left(\mathrm{QQ} \mid \mathrm{M}_{\mathrm{i}}\right)-\mathrm{P}\left(\mathrm{qq} \mid \mathrm{M}_{\mathrm{i}}\right)$

$\mathrm{c}_{\mathrm{d}}=\mathrm{P}\left(\mathrm{Qq} \mid \mathrm{M}_{\mathrm{i}}\right)$

em que: $\mathrm{P}\left(\mathrm{QQ} \mid \mathrm{M}_{\mathrm{i}}\right)=$ probabilidade condicional de os alelos do QTL serem homozigotos de origem da raça naturalizada brasileira Piau; $\mathrm{P}\left(\mathrm{qq} \mid \mathrm{M}_{\mathrm{i}}\right)$ = probabilidade condicional de os alelos do QTL serem homozigotos de origem da raça comercial; $\mathrm{P}\left(\mathrm{Qq} \mid \mathrm{M}_{\mathrm{i}}\right)$ = probabilidade condicional de os alelos do QTL serem heterozigotos.

O modelo anterior foi utilizado para estimar a regressão do fenótipo nos coeficientes $\mathrm{C}_{\mathrm{a}}$ e $\mathrm{C}_{\mathrm{d}}$, variando a posição do QTL a cada cM usando o mapa médio entre os dois sexos. Para cada posição, era calculada uma razão de verossimilhança (F), comparando-se o modelo que considera a presença do QTL (modelo completo) ao modelo sem o QTL (modelo reduzido). A curva dos valores da razão de verossimilhança (F) ao longo do cromossomo foi projetada em um gráfico, de modo que o ponto mais alto representa a provável posição do QTL. Os níveis de significância ( $\alpha=0,05$ ou 0,01$)$ "cromossome-wide" foram obtidos pelo teste de permutação (Churchill \& Doerge, 1994) para um total de 10.000 permutações para cada característica.

\section{Resultados e Discussão}

A distância média entre os marcadores foi de 38 cM cobrindo um intervalo de $152 \mathrm{cM}$ no cromossomo 4 de suínos. O tamanho do mapa está de acordo com a literatura e variou de 130,1 cM(USDA-MARC.2; Rohrer et al., 1996) a 165,0 cM (PiGMaP.1; Archibald et al., 1995). Não se observou mudança na ordem dos marcadores, o que está 
de acordo com outros estudos (Walling et al., 1998; Gerbens et al., 2000) (Tabela 3).

O conteúdo de informação polimórfica (PIC) quantifica a informação disponível para obtenção do genótipo do QTL em certa posição do cromossomo. Os baixos valores para o PIC para os marcadores pode estar relacionado ao baixo número de alelos segregando nesta população, o que pode ter sido provocado pelo pequeno número de animais fundadores (dois machos e 18 fêmeas). No entanto, de acordo com Botstein et al. (1980), os marcadores com PIC de 0,25 a 0,5 são classificados como moderadamente polimórficos e, portanto, estão aptos para utilização como marcadores em projetos de mapeamento genômico.

Os níveis de significância cromossômico a 5 e a $1 \%$ para os valores de F foram, respectivamente, 4,86 e 6,60 (Tabela 4).

Ao contrário de outros trabalhos em que foram encontrados vários QTL para as características de crescimento no cromossomo 4 de suínos (Bidanel et al., 2001; Knott et al., 2002; Geldermann et al., 2003), neste trabalho identificou-se apenas um QTL na população de estudo. O QTL identificado está envolvido no controle da característica P77 ( $<<0,01$, a nível cromossômico) e está próximo ao marcador S0217 (Tabela 4; Figura 1). Este QTL foi identificado em uma região do cromossomo 4 na qual Andersson et al. (1994) encontraram um QTL para taxa de crescimento e espessura de toucinho em uma população obtida do cruzamento do porco selvagem Europeu e suínos da raça Large White.

Marklund et al. (1999) confirmaram a presença deste QTL para taxa de crescimento e deposição de gordura quando utilizaram animais F2 do cruzamento realizado por Andersson et al. (1994) e os retrocruzaram com animais Large White. Esta região foi designada por esses autores como região FAT1. De acordo com Kim et al.(2006), os marcadores associados a maior crescimento também estão associados a menor deposição de gordura. Provavelmente existe um gene com efeito pleitrópico para essas duas características nesta região ou dois ou mais genes ligados estão se expressando.

De acordo com o modelo utilizado, os valores negativos para as estimativas dos efeitos aditivos indicam que o alelo herdado da raça naturalizada brasileira Piau foi responsável pela diminuição do peso aos 77 dias de idade. Esse resultado está de acordo com a maioria dos obtidos em outros trabalhos com cruzamentos de raças não melhoradas, principalmente a raça chinesa Meishan e o porco selvagem Europeu, em que os alelos herdados destas raças eram responsáveis pelo atraso no crescimento (Andersson et al., 1994: Bidanel et al., 2001). A raça naturalizada brasileira Piau se caracteriza por baixo desempenho, pequeno tamanho de leitegada e principalmente pelo grande acúmulo de

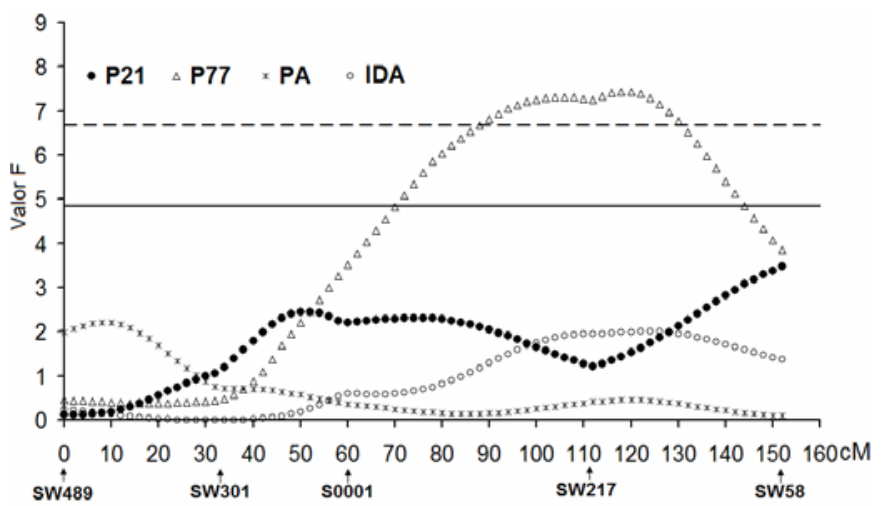

Figura 1 - Estimativas dos valores de F para as características peso aos 21 dias (P21), peso aos 77 dias (P77), peso ao abate (PA) e idade ao abate (IDA). As linhas horizontais indicam os níveis de significância ao longo do cromossomo para QTL significativo (5\%= linha contínua e a $1 \%$ = linha tracejada).

Tabela 4 - Verossimilhanças (F) máximas, suas posições (cM) nos QTL e respectivas estimativas dos efeitos aditivos e de dominância

\begin{tabular}{lccrr}
\hline Característica & Posição $^{1}$ (intervalo de confiança) & Fmáx & Aditivo ( \pm erro-padrão) & Dominância $( \pm$ erro-padrão) \\
\hline Número de tetas & 152 & 2,21 & $0,14 \pm 0,11$ & $0,30 \pm 0,17$ \\
Peso ao nascer (kg) & 0 & 2,55 & $-0,04 \pm 0,02$ & $-0,08 \pm 0,04$ \\
Peso aos 21 dias (kg) & 152 & 3,48 & $-0,15 \pm 0,14$ & $0,47 \pm 0,20$ \\
Peso aos 42 dias (kg) & 85 & 2,61 & $-0,54 \pm 0,32$ & $1,37 \pm 0,84$ \\
Peso aos 63 dias (kg) & 119 & 3,22 & $-1,03 \pm 0,47$ & $1,08 \pm 1,16$ \\
Peso aos 77 dias (kg) & $119(76-139)^{* *}$ & 7,44 & $-1,91 \pm 0,52$ & $0,70 \pm 1,27$ \\
Peso aos 105 dias (kg) & 87 & 4,80 & $-3,01 \pm 1,03$ & $-2,54 \pm 2,77$ \\
Consumo de ração (kg) & 9 & 2,20 & $-2,16 \pm 1,15$ & $-2,52 \pm 2,43$ \\
Ganho de peso (kg/dia) & 17 & 3,90 & $-0,08 \pm 0,02$ & $0,11 \pm 0,05$ \\
Conversão alimentar (75 aos 105 dias) & 0 & 4,74 & $-1,13 \pm 0,63$ & $2,83 \pm 1,21$ \\
Peso ao abate (kg) & 22 & 4,77 & $-0,27 \pm 0,1$ & $0,32 \pm 0,20$ \\
Idade ao abate (dias) & 124 & 2,00 & $2,40 \pm 1,25$ & $-0,68 \pm 2,90$ \\
\hline
\end{tabular}

${ }^{1}$ Provável posição do QTL.

** Significativo a 1\% ao nível cromossômico. 
gordura subcutânea (Guimarães \& Lopes, 2001). O efeito aditivo do alelo herdado da raça naturalizada brasileira Piau foi de $-1,91 \pm 0,52$, responsável por $9 \%$ da variabilidade fenotípica da característica. Desta forma, animais homozigotos para o alelo herdado da raça naturalizada Piau podem pesar aproximadamente $4 \mathrm{~kg}$ a menos aos 77 dias de idade em comparação aos homozigotos para os alelos herdados da raça comercial. Outros cromossomos desta mesma população continuam a ser estudados, assim como diferentes marcadores estão sendo incluídos no cromossomo 4, não apenas para melhorar a precisão de mapeamento, mas para confirmar a presença deste QTL para a característica de desempenho e crescimento.

O QTL encontrado para P77 confirma os resultados obtidos em outros trabalhos em que foi encontrado QTL associado ao crescimento em uma região similar do cromossomo 4 (Andersson et al., 1994; Wang et al., 1998; Paszek et al., 1999; Walling et al., 2000). Rohrer (2000), no entanto, não identificou nenhum QTL no cromossomo 4 para as características de crescimento quando utilizou animais oriundos do cruzamento entre Meishan e um composto de raças comerciais. Possivelmente os alelos deste gene responsáveis pelo controle destas características não estavam segregando na população.

Muráni et al. (2006), ao estudarem o polimorfismo no gene do hormônio que estimula a produção de Corticotropina (Corticotropin-releasing hormone - $\mathrm{CRH}$ ), localizado no SSC4, na região 4q13, identificaram a presença de uma troca nucleotídica $(\mathrm{G}>\mathrm{A})$ entre animais comerciais e uma população de suínos miniatura da Alemanha (DUMI). Esses autores observaram que os animais da população comercial tinham o alelo G fixado, enquanto os suínos miniatura eram heterozigotos e homozigotos para o alelo A. Segundo os autores, o alelo A foi responsável pelo retardamento no crescimento dos animais. A troca nucleotídica, no entanto, não estava associada a maior ou menor deposição de gordura (Muráni et al., 2006).

O hormônio corticotropina está envolvido no controle do balanço energético e controla o crescimento e a formação corporal; está localizado no braço longo do SSC4, assim como o marcador S0217; é candidato posicional para o QTL mapeado para característica de crescimento neste trabalho, e em outros já realizados (Knott et al., 1998; Bidanel et al., 2001; Varona et al., 2002). Uma vez que foi verificado QTL para crescimento em diferentes populações, seus efeitos devem ser investigados em populações comerciais como locos em potencial no uso da seleção assistida por marcadores (MAS). A confirmação do gene CRH como candidato em outras populações e a estimação de seus efeitos nas características de crescimento poderiam trazer benefícios para a indústria suinícola pela seleção de animais diretamente pelo genótipo.

\section{Conclusões}

Um QTL significativo foi evidenciado a nível cromossômico para a característica peso aos 77 dias (P77). Contudo, o intervalo de confiança onde se encontra o QTL ainda é muito grande e tem a presença de muitos genes. A utilização deste QTL na Seleção Assistida por Marcadores poderá ser feita após a confirmação em outras populações, o refino no mapeamento e a possível identificação da mutação causal e estimação de seus efeitos. Uma vez obtidas, estas informações genômicas serão de grande interesse para a indústria, pois possiblitarão a fixação ou a eliminação de alelos de genes responsáveis pelo controle de diversas características.

\section{Agradecimentos}

Ao pesquisador Marcos Vinícius G. B. Silva da EMBRAPA-CNPGL, pela colaboração, e ao Dr. Max Rothschild, coordenador do US Pig Genome Project, pela doação dos primers microssatélites.

\section{Literatura Citada}

ANDERSSON, L.; HALEY, C.S.; ELLEGREN, H. et al. Genetic mapping of quantitative trait loci for growth and fatness in pigs. Science, v.263, p.1771-1774, 1994.

ARCHIBALD, A.L.; HALEY, C.S.; BROWN, J.F. et al. The PiGMaP consortium linkage map of the pig (Sus scrofa). Mammalian Genome, v.6, p.157-175, 1995.

BAND, G.O.; GUIMARÃES, S.E.F.; LOPES, P.S. et al. Relationship between the Porcine Stress Syndrome gene and carcass and performance traits in F2 pigs resulting from divergent crosses. Genetics and Molecular Biology, v.28, n.1, p.92-96, 2005.

BIDANEL, J.P.; MILAN, D.; IANNUCCELLI, N. et al. Detection of quantitative trait loci for growth and fatness in pigs. Genetic Selection Evolution, v.33, p.289-309, 2001.

BINADEL, J.P.; ROTHSCHILD, M. Current status of quantitative trait locus mapping in pigs. Pig News and Information, v.23, p.39-54, 2002

BOTSTEIN, D.; WHITE, R.L.; SKOLNICK, M. et al. Construction of a genetic linkage map in man using restriction fragment length polymorphisms. American Journal Human Genetics, v.32, p.314-331, 1980.

CHURCHILL, G.A.; DOERGE, R.W. Empirical threshold values for quantitative trait mapping. Genetics, v.138, p.963-971, 1994.

DEKKERS, J.C.M.; HOSPITAL, F. The use of molecular genetics in improvement of agricultural populations. Nature Reviews Genetics, v.3, p.22-32, 2002.

GELDERMANN, H. Investigations on inheritance on quantitative characters in animals by gene markers. I. Methods. Theoretical and Applied Genetics, v.46, p.319-330, 1975 
GELDERMANN, H.; MULLER, E.; MOSER, G. et al. Genome-wide linkage and QTL mapping in porcine F2 families generated from Piétrain, Meishan and Wild Boar crosses. Journal Animal Breeding and Genetics, v.120, p.363-393, 2003.

GERBENS, F.; DE KONING, D.J.; HARDERS, F.L. et al. The effect of adipocyte and heart fatty acid-binding protein genes on intramuscular fat and backfat content in Meishan crossbred pigs. Journal of Animal Science, v.78, n.3, p.552-559, 2000.

GREEN. P.; FALLS, K.; CROOKS, S. Documentation for CRIMAP. St. Louis: Washington University School of Medicine, 1990.

GUIMARÃES, S.E.F.; LOPES, P.S. Uso de recursos genéticos nativos no mapeamento genético de suínos. Ação Ambiental, Ano III, n.15, p.27-28, 2001.

HALEY, C.S.; KNOTT, S.A.; ELSEN, J.M. Mapping quantitative trait loci in crosses between outbred lines using least squares. Genetics, v.36, p.1195-1207, 1994.

HENSHALL, J.M.; GODDARD, M.E. Comparison of marker assisted selection using mixed model (BLUP) and mixed model with a test for the QTL. Dubbo: Association of Advancement of Animal Breeding and Genetics, 1997. p.217-221.

KIM, K.S.; LEE, J.J.; SHIN, H.Y. et al. Association of melanocortin 4 receptor (MC4R) and high mobility group AT-hook 1 (HMGA1) polymorphisms with pig growth and fat deposition traits. Animal Genetics, v.37, p.419-421, 2006.

KNOTT, S.A.; MARKLUND, L.; HALEY, C.S. et al. Multiple marker mapping of quantitative trait loci in a cross between outbred wild boar and Large White pigs. Genetics, v.148, p.1069-1080, 1998.

KNOTT, S.A.; NYSTROM, P.E.; ANDERSSON-EKLUND, L. et al. Approaches to interval mapping of QTL in a multigeneration pedigree: the example of porcine chromosome 4. Animal Genetics, v.33, p.26-32, 2002.

LANDE, R. The minimum number of genes contributing to quantitative variation between and within populations. Genetics, v.121, p.185-199, 1981

MARKLUND, L.; NYSTROM, P.E.; STREN, S. et al. Confirmed quantitative trait loci for fatness and growth on pig chromosome 4. Heredity, v.82, p.134-141, 1999.

MARSHALL, T.C.; SLATE, J.; KRUUK, L. et al. Statistical confidence for likelihood-based paternity inference in natural populations. Molecular Ecology, v.7, p.639-655, 1998.
MILLER, S.A.; DYKES, D.D.; POLESKY, H.F. A simple salting out procedure for extracting DNA from human nucleated cells. Nucleic Acids Research, v.16, p.1215, 1988.

MURÁNI, E.; MURÁNIOVÁ, M.; PONSUKSILI, S. et al. Molecular characterization and evidencing of the porcine CRH gene as a functional-positional candidate for growth and body composition. Biochemical and Biophysical Research Communications, v.342, p.394-405, 2006.

PASZEK, A.A.; WILKIE, P.J.; FLICKINGER, G.H. et al. Interval mapping of growth in divergent swine cross. Mammalian Genome, v.10, p.117-122, 1999.

PEREIRA, J.C.C. Melhoramento genético aplicado à produção animal. 3.ed. Belo Horizonte: FEPMVZ Editora, 2001. 555p.

PÉREZ-ENCISO, M.; CLOP, A.; NOGUERA, J.L. et al. A QTL on pig chromosome 4 affects fatty acid metabolism: Evidence from a Iberian by Landrace intercross. Journal of Animal Science, v.78, p.2525-2531, 2000.

ROHRER, G.A. Identification of quantitative trait loci affecting birth characters and accumulation of backfat and weight in a Meishan-White Composite resource population. Journal Animal Science, v.78, p.2547-2553, 2000.

ROHRER, G.A.; ALEXANDER, L.J.; HU, Z. et al. A comprehensive map of the porcine genome. Genome Research, v.6, p.371-391, 1996.

SEATON, G.; HALEY, C.S.; KNOTT, S.A. et al. [2002]. QTL express: mapping quantitative trait loci in simple and complex pedigrees. Bioinformatics, v.18, n.2, p.339-340, 2002. Disponível em: <http://qtl.cap.ed.ac.uk>. Acesso em: 3/1/2007.

VARONA, L.; OVILO, A.C.; NOGUERA, J.L. et al. QTL mapping for growth and carcass traits in a Iberian by Landrace pig intercross: additive, dominant and epistatic affects. Genetics Research Cambrige, v.80, p.145-154, 2002.

WALLING G.A.; ARCHIBALD, A.L.; CATTERMOLE, J.A. et al. Mapping of quantitative trait loci on porcine chromosome 4. Animal Genetics, v.29, p.415-424, 1998

WALLING, G.A.; VISSCHER, P.M.; ANDERSSON, L. et al. Combined analyses of data from quantitative trait loci mapping studies: chromosome 4 effects on porcine growth and fatness. Genetics, v.155, p.1369-1378, 2000.

WANG, L.; YU, T.P.; TUGGLE, C.K. et al. A direct search for quantitative trait loci on chromosomes 4 and 7 in pigs. Journal of Animal Science, v.76, p.2560-2567, 1998. 